\title{
Codimension three bifurcation of streamline patterns close to a no-slip wall: A topological description of boundary layer eruption
}

\author{
Balci, A.; Andersen, M.; Thompson, M. C.; Brøns, M.
}

Published in:

Physics of Fluids

DOI:

10.1063/1.4921527

Publication date:

2015

\section{Document Version}

Publisher's PDF, also known as Version of record

Citation for published version (APA):

Balci, A., Andersen, M., Thompson, M. C., \& Brøns, M. (2015). Codimension three bifurcation of streamline patterns close to a no-slip wall: A topological description of boundary layer eruption. Physics of Fluids, $27(5)$, - . https://doi.org/10.1063/1.4921527

\section{General rights}

Copyright and moral rights for the publications made accessible in the public portal are retained by the authors and/or other copyright owners and it is a condition of accessing publications that users recognise and abide by the legal requirements associated with these rights.

- Users may download and print one copy of any publication from the public portal for the purpose of private study or research.

- You may not further distribute the material or use it for any profit-making activity or commercial gain.

- You may freely distribute the URL identifying the publication in the public portal.

\section{Take down policy}

If you believe that this document breaches copyright please contact rucforsk@kb.dk providing details, and we will remove access to the work immediately and investigate your claim. 


\section{AIP | Physics of}

Codimension three bifurcation of streamline patterns close to a no-slip wall: A topological description of boundary layer eruption

A. Balci, M. Andersen, M. C. Thompson, and M. Brøns

Citation: Physics of Fluids (1994-present) 27, 053603 (2015); doi: 10.1063/1.4921527

View online: http://dx.doi.org/10.1063/1.4921527

View Table of Contents: http://scitation.aip.org/content/aip/journal/pof2/27/5?ver=pdfcov

Published by the AIP Publishing

\section{Articles you may be interested in}

Investigation of the separated region ahead of three-dimensional protuberances on plates and cones in hypersonic flows with laminar boundary layers

Phys. Fluids 26, 126101 (2014); 10.1063/1.4902400

Open-loop control of noise amplification in a separated boundary layer flow

Phys. Fluids 25, 124106 (2013); 10.1063/1.4846916

Particle image velocimetry measurements of the interaction of synthetic jets with a zero-pressure gradient laminar boundary layer

Phys. Fluids 22, 063603 (2010); 10.1063/1.3432133

Flow past a square cylinder with an angle of incidence

Phys. Fluids 22, 043603 (2010); 10.1063/1.3388857

Streamline patterns and their bifurcations near a wall with Navier slip boundary conditions

Phys. Fluids 18, 083102 (2006); 10.1063/1.2337660

SHARE

your expertise in simulation

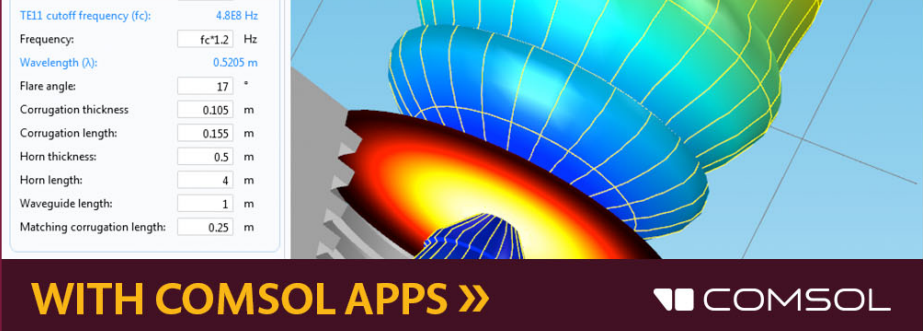
\begin{tabular}{lr}
\hline Input waveguide cross pol. ratio: & $17.657 \%$ \\
Output aperture cross pol. ratio: & $3.025 \%$
\end{tabular} 


\title{
Codimension three bifurcation of streamline patterns close to a no-slip wall: A topological description of boundary layer eruption
}

\author{
A. Balci, ${ }^{1, a)}$ M. Andersen, ${ }^{1,2, b)}$ M. C. Thompson, ${ }^{3, c}$ ) and M. Brøns ${ }^{1, d)}$ \\ ${ }^{1}$ Department of Applied Mathematics and Computer Science, Technical University \\ of Denmark, 2800 Kongens Lyngby, Denmark \\ ${ }^{2}$ Department of Science, Systems and Models, Roskilde University, 4000 Roskilde, Denmark \\ ${ }^{3}$ Fluids Laboratory for Aeronautical and Industrial Research (FLAIR), Department \\ of Mechanical and Aerospace Engineering, Monash University, \\ Melbourne, Victoria 3800, Australia
}

(Received 30 July 2014; accepted 4 May 2015; published online 29 May 2015)

\begin{abstract}
A vortex close to a no-slip wall gives rise to the creation of new vorticity at the wall. This vorticity may organize itself into vortices that erupt from the separated boundary layer. We study how the eruption process in terms of the streamline topology is initiated and varies in dependence of the Reynolds number $R e$. We show that vortex structures are created in the boundary layer for $R e$ around 600 , but that these disappear again without eruption unless $R e>1000$. The eruption process is topologically unaltered for $R e$ up to 5000. Using bifurcation theory, we obtain a topological phase space for the eruption process, which can account for all observed changes in the Reynolds number range we consider. The bifurcation diagram complements previously analyzes such that the classification of topological bifurcations of flows close to no-slip walls with up to three parameters is now complete. (C) 2015 AIP Publishing LLC. [http://dx.doi.org/10.1063/1.4921527]
\end{abstract}

\section{INTRODUCTION}

A vortex convected close to a no-slip wall induces a viscous response from the wall boundary layer. For a sufficiently high Reynolds number, vorticity from the boundary layer is ejected into the surrounding fluid, resulting in secondary vortex structures. This phenomenon has been denoted eruption of the boundary layer, ${ }^{1,2}$ unsteady separation, ${ }^{3,4}$ or bursting, ${ }^{5}$ and it occurs for a wide range of Reynolds numbers. ${ }^{6,7}$ The interaction between concentrated vorticity in a fluid and the vorticity created at a no-slip wall occurs in many important settings. In dynamic stall of an unsteady airfoil, the vortex created at the leading edge plays a key role in generating the transition to separated flow. ${ }^{8,9}$ A vortex wake created by the flow around a bluff body may interact with a wall or a free surface and give rise to secondary vortices. ${ }^{10-13}$ This is also the case when vortices, solid bodies, or jets impinge directly on a wall. ${ }^{14-17}$

Here, we are concerned with a precise and formal description of the qualitative changes in the flow structure that occur during the eruption process and how the sequence of these transitions depends on the Reynolds number. Our starting point is the analysis by Kudela and Malecha ${ }^{1,2}$ who showed numerically how the streamline pattern changes through several stages as a vortex erupts from the boundary layer. We will provide further numerical simulations to understand the process in more detail and also provide a theoretical framework to establish the possible topological changes in the streamline pattern as time and Reynolds number vary.

\footnotetext{
${ }^{a)}$ Electronic mail: adbal@dtu.dk

b) Electronic mail: moan@ ruc.dk

c) Electronic mail: mark.thompson@monash.edu

d) Electronic mail: mobr@dtu.dk
} 
No generally accepted or generally applicable definition of spatial structures in fluid flows is available, and even for a fundamental object such as a vortex, many definitions have been suggested. ${ }^{18-20}$ Possibly, the most elementary approach to the identification of structures in flows is to consider the topology of the streamlines, that is, the trajectories of the system of ordinary differential equations in dimensions two or three,

$$
\dot{\mathbf{x}}=\mathbf{v}(\mathbf{x}),
$$

where $\mathbf{v}$ is the instantaneous velocity field. From the qualitative theory of differential equations, it is known that the phase portrait of an autonomous system like (1) is primarily organized by the critical points defined by $\mathbf{v}=\mathbf{0}$ and the stable and unstable manifolds of these points which form dividing streamlines and streamsurfaces. The analysis of streamline topology has a long history in fluid mechanics with pioneering work by Dean ${ }^{21}$ and Legendre ${ }^{22-24}$ followed by many others. ${ }^{25-28}$ If parameters such as the Reynolds number or geometric properties are varied, bifurcation theory can be applied to characterize the changes in the streamline pattern that may arise. For unsteady flows, time can also be considered a parameter in system (1). A general bifurcation theory for streamline patterns has been developed by several authors ${ }^{28-33}$ and many applications to specific flow problems such as vortex breakdown, ${ }^{34,35}$ driven cavities, ${ }^{36-38}$ the cylinder wake, ${ }^{39,40}$ and peristaltic flows ${ }^{41}$ are available.

The analysis of topological bifurcations consists in identifying degenerate streamline patterns and their unfoldings, that is, parametrized families of velocity fields which can represent all possible perturbations of the given degenerate pattern. By a series of coordinate transformations, one tries to obtain a special unfolding, denoted a normal form, which contains as few free parameters as possible. The number of parameters is the codimension of the degeneracy. The parameter space is a bifurcation diagram which is partitioned by bifurcation curves and surfaces into regions of different flow topologies. Such a bifurcation diagram constitutes a topological phase space for a class of flow problems.

We organize our analysis as follows: In Sec. II, we report detailed numerical simulations of the vortex-driven flow for $R e$ up to 5000 and establish all the topological bifurcations that are relevant. In Sec. III, we first note that none of the topological phase spaces which are available in the literature can account for all the observed bifurcations in the eruption process and thereafter proceed to establish and analyze the relevant normal form which has codimension three. In Sec. IV, we verify that the normal form does in fact cover all the observed bifurcations, and in Sec. V, we draw conclusions and provide an outlook.

\section{TOPOLOGICAL BIFURCATIONS IN BOUNDARY LAYER ERUPTION}

From numerical computations, Kudela and Malecha ${ }^{1,2}$ have identified various changes in the topology of the streamlines during the eruption process, when described in a coordinate system fixed to the wall. The configuration is shown in Fig. 1. At $t=0$, a Gaussian vortex with core radius $a$ and negative circulation $-\Gamma$ is placed a distance $d$ from a flat wall, and an image vortex is placed

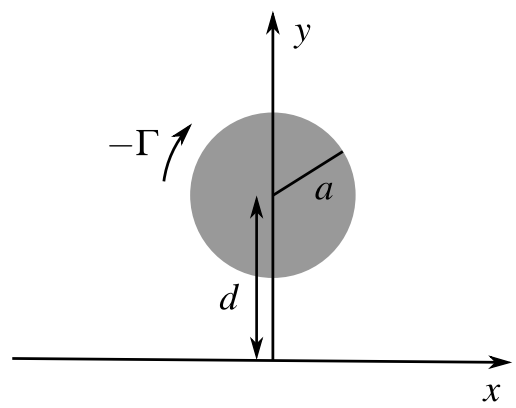

FIG. 1. Initial configuration of the eruption process. A Gaussian vortex with core radius $a$ and negative circulation $-\Gamma$ is placed at $(0, d)$. 

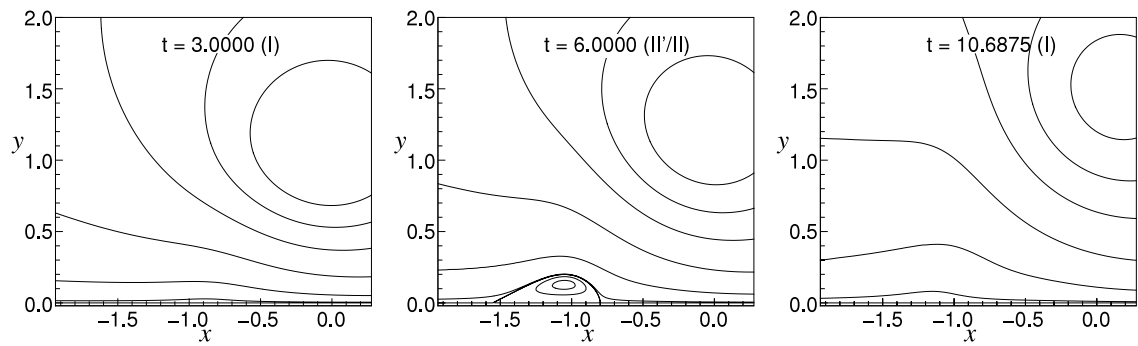

FIG. 2. Numerically obtained streamlines for the vortex-induced flow for $R e=200$. In each panel, the dimensionless time and the topological classification according to the bifurcation diagram in Fig. 11 are shown.

below the wall. This is then evolved for a short relaxation time with a free-slip wall boundary to smooth out transient oscillations, allowing the flow to adjust. ${ }^{42}$ When the initial oscillations are reduced, the wall boundary condition is instantaneously switched to a no-slip wall and the computation is continued. The equations were solved in a coordinate system where the vortices are initially at rest. The equations are non-dimensionalized by the length scale $L=d$ and the time scale $T=2 \pi a d / \Gamma$. The Reynolds number is defined by $R e=L^{2} / T \nu$. The flow equations are solved by a well-established finite-element code using GLL quadrature and Lagrange polynomials. ${ }^{43-45}$ Standard domain and resolution studies were performed to ensure the reliability of predictions, and very good agreement with the results by Kudela and Malecha ${ }^{2}$ is obtained. For more details on the computational aspects, see Ref. 46.

In Figs. 2-6, we show representative sequences of streamline patterns for increasing values of $R e$. Shortly after the initiation of the flow, the main vortex appears as closed streamlines encircling a critical point (not displayed) located at $(0,1)$ at $t=0$. At later times, the pictures focus on structures close to the wall such that the main vortex is outside the plotting window. Each streamline pattern is classified according to its topology following the theory we develop in Sec. III.

For $R e=200$, Fig. 2, a separation zone occurs at the wall which grows and then shrinks and disappears again. Not further changes in topology occur, and there is consequently no eruption. For $R e=600$, Fig. 3, a separation zone is again created, but rather than shrinking while attached to the wall it pinches off, creating a saddle point in the fluid. The dividing streamlines of the saddle enclose a region with closed streamlines around a critical point of center type. This can be considered as a vortex structure which is not yet erupted. However, soon after the creation, the vortex structure shrinks and disappears as the saddle and the center merge in a saddle-center bifurcation before it has left the boundary layer.

For $R e=800$, Fig. 4, a saddle-center bifurcation occurs inside the attached separation zone. This creates a figure-eight structure visible at $t=10.3125$. Shortly thereafter, another saddle-center bifurcation occurs where the lower center and the saddle disappear, leaving only a single center inside the separation zone. The rest of the process is identical to that at $R e=600$.

For $R e=1000$ and up to 5000, eruption takes place following the sequence of events which we show in Fig. 5. As for $R e=800$, a figure-eight is created inside the separation zone $(t=13.3225)$, but now the top center and the saddle pinch off in a global bifurcation, leaving a separation zone attached to the wall and an erupted vortex structure $(t=13.89750)$. The separation zone shrinks
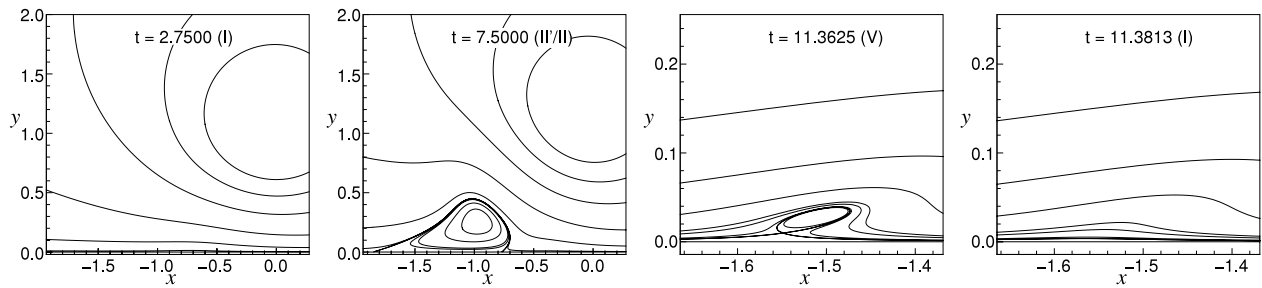

FIG. 3. As Fig. 2, but for $R e=600$. 

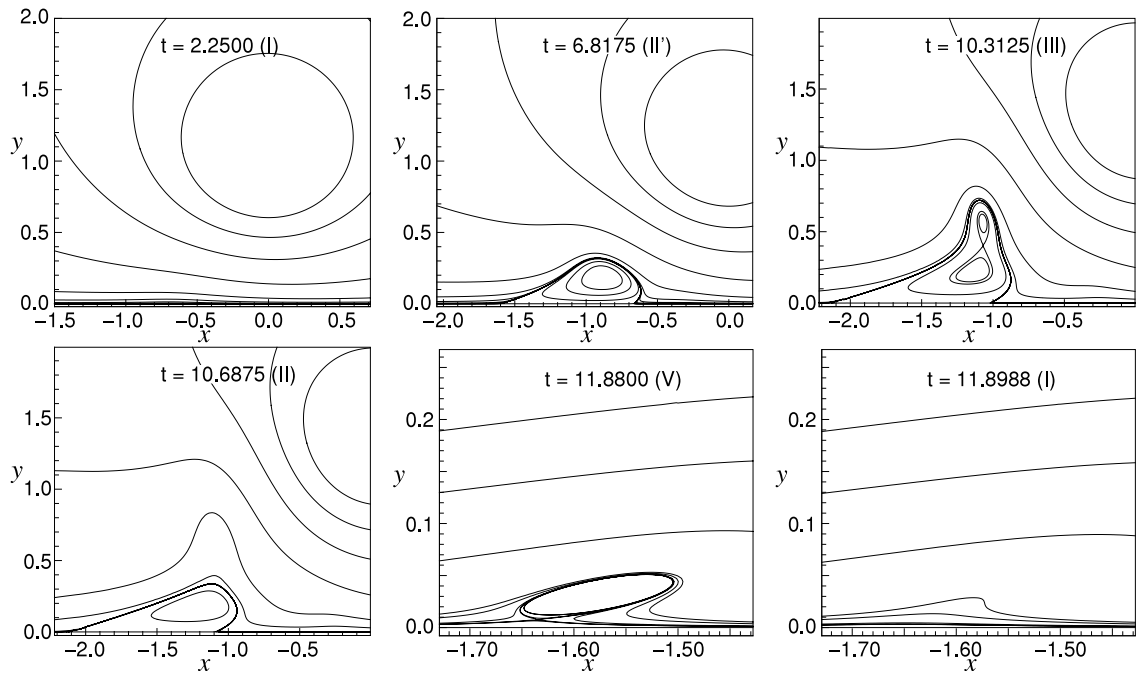

FIG. 4. As Fig. 2, but for $R e=800$.

and disappears, while the erupted vortex leaves the boundary layer as it rotates around the main vortex, much like two point vortices of opposite sign in an ideal fluid would do.

At later stages, further topological changes may occur if $R e$ is sufficiently high. We illustrate this for $R e=5000$ in Fig. 6. Here, an attached separation zone is again created, and a little later, a vortex structure appears above it in a saddle-center bifurcation. It does not erupt but merges with the separation zone which subsequently disappears in a few bifurcations. For higher values of $R e$, these secondary topological changes may lead to eruption ${ }^{2}$ and generate more complex streamline patterns which are outside the scope of the present study.

\section{TOPOLOGICAL ANALYSIS}

\section{A. The setting of the analysis}

We consider two-dimensional incompressible flow close to a flat no-slip wall. The local structure of the streamlines close to a point which we take to be the origin can be found from a Taylor
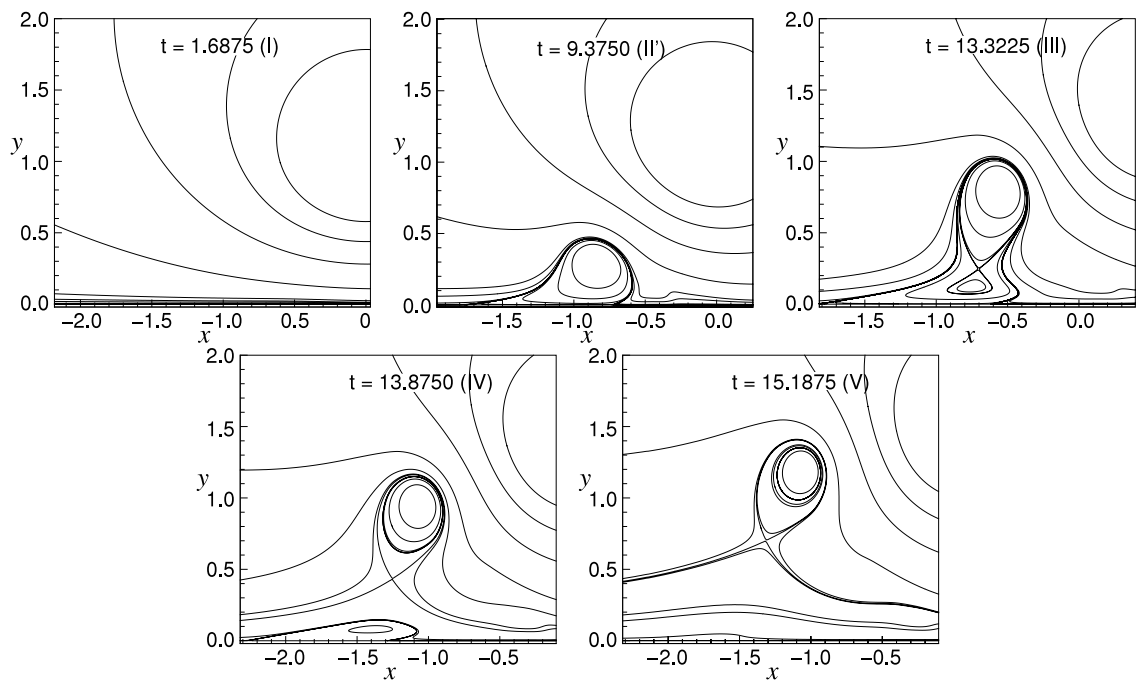

FIG. 5. As Fig. 2, but for $R e=5000$, early stages 


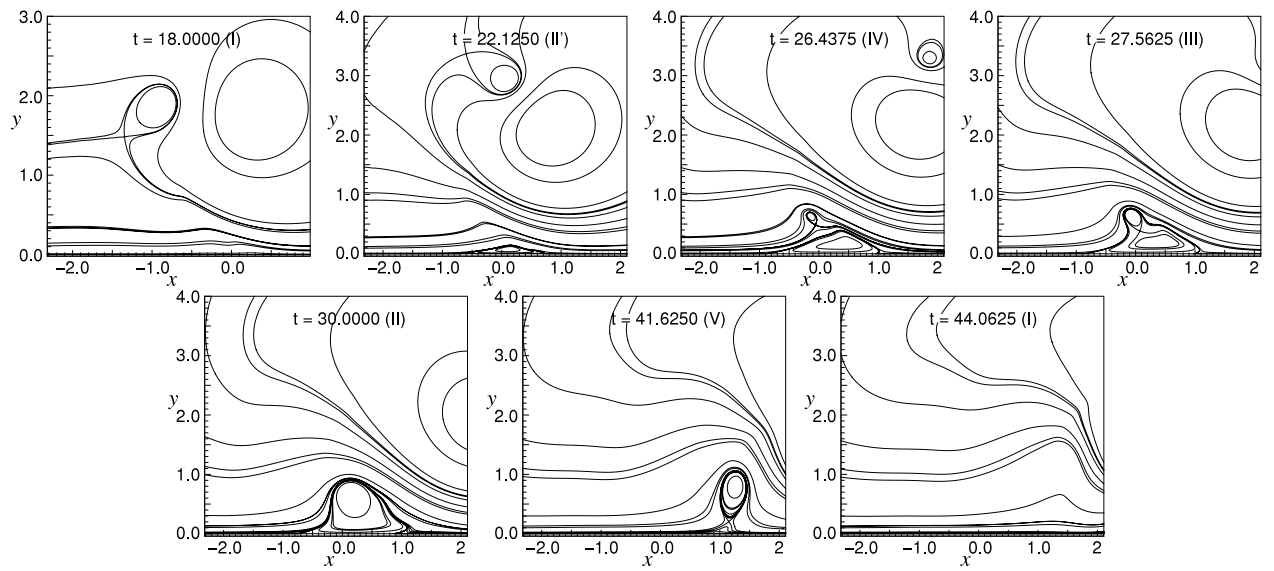

FIG. 6. As Fig. 2, but for $R e=5000$, later stages.

expansion of the stream function,

$$
\psi=y^{2} \sum_{n, m=0}^{\infty} a_{n, m+2} x^{n} y^{m} .
$$

Here, the wall is given by $y=0$ and the factor $y^{2}$ appears as a consequence of the no-slip boundary condition. The velocity components are given by

$$
u=\frac{\partial \psi}{\partial y}, \quad v=-\frac{\partial \psi}{\partial x},
$$

but rather than considering differential equations (1), a time-scaled version where a common factor $y$ is eliminated is normally used,

$$
\dot{x}=\hat{u}=\frac{u}{y}=2 a_{0,2}+2 a_{1,2} x+3 a_{0,3} y+\cdots, \quad \dot{y}=\hat{v}=\frac{v}{y}=-a_{1,2} y+\cdots .
$$

This system has a critical point at the origin if $a_{0,2}=0$, and the type of the point is given by the eigenvalues of the Jacobian matrix,

$$
J=\left(\begin{array}{cc}
2 a_{1,2} & 3 a_{0,3} \\
0 & -a_{1,2}
\end{array}\right)
$$

If $a_{1,2} \neq 0$, the eigenvalues are real and non-zero, such that the critical point is a regular point of separation or attachment. If, however, $a_{1,2}=0$, the critical point is degenerate, and a small variation of the coefficients $a_{n, m}$ may result in a qualitative change of the local streamline pattern, that is, a bifurcation. A bifurcation analysis is efficiently approached by obtaining a normal form, where as many higher-order terms as possible in (4) are removed by non-linear coordinate transformations. The number of bifurcation parameters which remain in the normal form is the co-dimension of the degenerate critical point. Under the non-degeneracy condition $a_{0,3} \neq 0$, normal forms and bifurcation diagrams of co-dimension up to three have been obtained in Refs. 28, 31, and 32. This is known as the simple case, while the case $a_{0,3}=0$ is non-simple. The most basic non-simple situation has co-dimension two under the non-degeneracy conditions

$$
a_{2,2} \neq 0, \quad \tilde{a}_{0,4}=a_{0,4}-\frac{a_{1,3}{ }^{2}}{4 a_{2,2}} \neq 0,
$$

see Refs. 28 and 31. Two different bifurcation diagrams result from this normal form, depending on the sign $\sigma$ of $\tilde{a}_{0,4} / a_{2,2}$. See, e.g., Fig. 11 in Ref. 31. For $\sigma>0$, the pinching off of a vortex from the wall as it occurs in Figs. 3 and 4 is possible. When $\sigma<0$, the pinching off of a vortex from a figure-eight inside a separation zone attached to the wall is possible. This is the main bifurcation leading to eruption as shown in Fig. 5, so to get a complete description of all the bifurcations 
in the vortex-driven flow, a single normal form allowing different signs of $\sigma$ is needed. Hence, non-degeneracy conditions (6) must be broken.

The case where the first condition is violated, together with other non-degeneracy conditions,

$$
a_{0,2}=a_{1,2}=a_{0,3}=a_{2,2}=0, \quad a_{1,3} \neq 0, \quad a_{3,2} \neq 0,
$$

has co-dimension three. ${ }^{37,40}$ However, it cannot account for the bifurcations we consider here.

The other case,

$$
a_{0,2}=a_{1,2}=a_{0,3}=\tilde{a}_{0,4}=0, \quad a_{2,2} \neq 0,
$$

has, to our knowledge, not been analyzed before. In the rest of this section, we will obtain the normal form and the bifurcation diagram associated with this degeneracy under a further non-degeneracy condition of the form $\tilde{a}_{0,5} \neq 0$, where $\tilde{a}_{0,5}$, given in (10c), is a parameter which appears in the course of the analysis. In Sec. IV, we will show that the bifurcation diagram is the relevant one for the vortex-driven flow. With this analysis, all topological bifurcation diagrams for flows close to a no-slip wall with co-dimension up to three have been obtained.

\section{B. Normal form for the degenerate case}

In this section, we perform a series of coordinate transformations in an attempt to simplify stream function (2) assuming conditions (8).

Following Hartnack, ${ }^{31}$ we introduce the new variable $\xi=x+\frac{a_{1,3}}{2 a_{2,2}} y$ to eliminate the term $a_{1,3} x y^{3}$. As a consequence of the assumption $\tilde{a}_{0,4}=0$, the streamfunction then reads

$$
\psi=y^{2}\left(a_{2,2} \xi^{2}+a_{3,2} \xi^{3}+\tilde{a}_{2,3} \xi^{2} y+\tilde{a}_{1,4} \xi y^{2}+\tilde{a}_{0,5} y^{3}+O\left(|\xi, y|^{4}\right)\right),
$$

where

$$
\begin{aligned}
& \tilde{a}_{2,3}=a_{2,3}-\frac{3 a_{3,2} a_{1,3}}{2 a_{2,2}}, \\
& \tilde{a}_{1,4}=a_{1,4}-\frac{a_{2,3} a_{1,3}}{a_{2,2}}+\frac{3 a_{3,2} a_{1,3}{ }^{2}}{4 a_{2,2}{ }^{2}}, \\
& \tilde{a}_{0,5}=a_{0,5}-\frac{a_{1,4} a_{1,3}}{2 a_{2,2}}+\frac{a_{2,3} a_{1,3}}{4 a_{2,2}{ }^{2}}-\frac{a_{3,2} a_{1,3}{ }^{3}}{8 a_{2,2}{ }^{3}} .
\end{aligned}
$$

Further simplifications can be obtained from non-linear coordinate transformations. We define a near-identity transformation such that the wall $y=0$ is mapped to $\eta=0$ by

$$
\xi=\chi+r_{2,0} \chi^{2}+r_{1,1} \chi \eta+r_{0,2} \eta^{2}, \quad y=\eta+s_{1,1} \chi \eta+s_{0,2} \eta^{2} .
$$

This transforms the stream function into

$$
\begin{aligned}
\psi & \eta^{2}\left(a_{2,2} \chi^{2}+\left(2 a_{2,2} r_{2,0}+2 s_{1,1} a_{2,2}+a_{3,2}\right) \chi^{3}\right. \\
& \left.+\left(2 a_{2,2} r_{1,1}+2 s_{0,2} a_{2,2}+\tilde{a}_{2,3}\right) \chi^{2} \eta+\left(2 a_{2,2} r_{0,2}+\tilde{a}_{1,4}\right) \chi \eta^{2}+\tilde{a}_{0,5} \eta^{3}+O\left(|\chi, \eta|^{4}\right)\right) .
\end{aligned}
$$

The terms $\chi^{3} \eta^{2}, \chi^{2} \eta^{3}$, and $\chi \eta^{4}$ can be eliminated by choosing $r_{2,0}=0, r_{1,1}=0, r_{0,2}=-\frac{\tilde{a}_{1,4}}{2 a_{2,2}}$, $s_{1,1}=-\frac{a_{3,2}}{2 a_{2,2}}$, and $s_{0,2}=-\frac{\tilde{a}_{2,3}}{2 a_{2,2}}$ and we get

$$
\psi=\eta^{2}\left(a_{2,2} \chi^{2}+\tilde{a}_{0,5} \eta^{3}+O\left(|\chi, \eta|^{4}\right)\right) .
$$

Finally, we scale time by dividing the stream function by $\tilde{a}_{0,5}$ and scale $\chi$ by substituting $\chi=$ $\sqrt{\mid \frac{\tilde{a}_{0,5} \mid}{a_{2,2} \mid}} \bar{x}$ and obtain

$$
\psi=\eta^{2}\left(\sigma \bar{x}^{2}+\eta^{3}+O\left(|\bar{x}, \eta|^{4}\right)\right),
$$

where

$$
\sigma=\frac{a_{2,2}}{\tilde{a}_{0,5}}\left|\frac{\tilde{a}_{0,5}}{a_{2,2}}\right|= \begin{cases}+1 & \text { for } a_{2,2} / \tilde{a}_{0,5}>0 \\ -1 & \text { for } a_{2,2} / \tilde{a}_{0,5}<0\end{cases}
$$


(a)

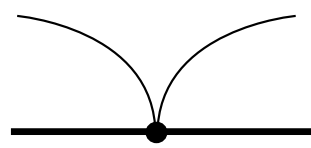

(b)

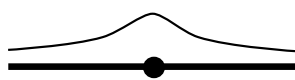

FIG. 7. Streamline patterns close to the degenerate critical point for normal form (14). (a): $\sigma=-1,(\mathrm{~b}): \sigma=1$.

and we have assumed $\tilde{a}_{0,5} \neq 0$. To analyse the topology of the flow close to the critical point $(0,0)$ of (14), we look for possible separatrices (dividing streamlines) by solving $\psi=0$. Disregarding the $O$-term, the solutions are

$$
\eta=0, \quad \eta=\left(-\sigma \bar{x}^{2}\right)^{1 / 3},
$$

where the first solution corresponds to the wall and the latter forms a cusp which is in the fluid domain $\eta>0$ only if $\sigma=-1$. The two possible streamline patterns are shown in Fig. 7 .

It is interesting to note that stream function (9) cannot occur in a steady flow. From the steady Navier-Stokes equations, further conditions on the coefficients appear which ensure ${ }^{31}$ that $\tilde{a}_{0,4}<0$. Hence, the analysis of this paper is relevant only for unsteady flows.

\section{Unfolding of the degenerate case}

The degenerate case is structurally unstable. Small perturbations of the parameters away from the degenerate value may result in different streamline patterns. Following the approach from Sec. III B, we will now derive a normal form to simplify the bifurcation analysis. We define again the new variable $\xi=x+\frac{a_{1,3}}{2 a_{2,2}} y$ and substituting this into (2), we get

$\psi=y^{2}\left(\epsilon_{1}+\epsilon_{2} \xi+\epsilon_{3} y+\epsilon_{4} y^{2}+a_{2,2} \xi^{2}+a_{3,2} \xi^{3}+\tilde{a}_{2,3} \xi^{2} y+\tilde{a}_{1,4} \xi y^{2}+\tilde{a}_{0,5} y^{3}+O\left(|\xi, y|^{4}\right)\right)$,

where

$$
\epsilon_{1}=a_{0,2}, \quad \epsilon_{2}=a_{1,2}, \quad \epsilon_{3}=a_{0,3}-\frac{a_{1,3} a_{1,2}}{2 a_{2,2}}, \quad \epsilon_{4}=\tilde{a}_{4}=a_{0,4}-\frac{a_{1,3}{ }^{2}}{4 a_{2,2}}
$$

are small (bifurcation) parameters and $\tilde{a}_{2,3}, \tilde{a}_{1,4}, \tilde{a}_{0,5}$ are given in (10).

To further simplify the stream function, we apply the near-identity transformation

$$
\xi=\chi+r_{0,2} \eta^{2}, \quad y=\eta+s_{1,1} \chi \eta+s_{0,2} \eta^{2} .
$$

As in the degenerate case, we aim at removing the $\chi^{3} y^{2}, \chi^{2} y^{3}$, and $\chi y^{4}$-terms. The coefficients for these terms in the transformed stream function are

$$
\begin{aligned}
f\left(\epsilon_{2}, s_{1,1}\right) & =s_{1,1}{ }^{2} \epsilon_{2}+a_{3,2}+2 s_{1,1} a_{2,2}, \\
g\left(\epsilon_{2}, \epsilon_{3}, s_{0,2}\right) & =2 s_{0,2} a_{2,2}+2 s_{1,1} s_{0,2} \epsilon_{2}+\tilde{a}_{2,3}+3 s_{1,1}^{2} \epsilon_{3}, \\
h\left(\epsilon_{2}, \epsilon_{3}, \epsilon_{4}, r_{0,2}\right) & =6 s_{1,1} \epsilon_{3} s_{0,2}+4 s_{1,1} r_{0,2} \epsilon_{2}+4 \epsilon_{4} s_{1,1}+\tilde{a}_{1,4}+2 a_{2,2} r_{0,2}+s_{0,2}{ }^{2} \epsilon_{2} .
\end{aligned}
$$

Since

$$
f\left(0,-\frac{a_{3,2}}{2 a_{2,2}}\right)=0, \quad \frac{\partial f}{\partial s_{1,1}}\left(0,-\frac{a_{3,2}}{2 a_{2,2}}\right)=2 a_{2,2} \neq 0,
$$

it follows from the implicit function theorem that there exists a function $s_{1,1}\left(\epsilon_{2}\right)$ with $s_{1,1}(0)=$ $-\frac{a_{3,2}}{2 a_{2,2}}$ such that $f\left(\epsilon_{2}, s_{1,1}\left(\epsilon_{2}\right)\right)=0$, for $\epsilon_{2}$ sufficiently small. Similarly, we find functions $s_{0,2}\left(\epsilon_{2}, \epsilon_{3}\right)$ and $r_{0,2}\left(\epsilon_{2}, \epsilon_{3}, \epsilon_{4}\right)$ which solve $g=0$ and $h=0$, respectively. Again, we only use the assumption $a_{2,2} \neq 0$ and the implicit function theorem. This yields the stream function

$$
\psi=\eta^{2}\left(\epsilon_{1}+\hat{\epsilon}_{2} \chi+\hat{\epsilon}_{3} \eta+\hat{a}_{2,2} \chi^{2}+\hat{\epsilon}_{4} \chi \eta+\hat{\epsilon}_{5} \eta^{2}+\hat{a}_{0,5} \eta^{3}\right),
$$


where the $\hat{\epsilon}$ are transformed small parameters and $\hat{a}_{2,2}=\tilde{a}_{2,2}+O(\hat{\epsilon}), \hat{a}_{0,5}=\tilde{a}_{0,5}+O(\hat{\epsilon})$. Further, by a scaling of the time by dividing the stream function by $\hat{a}_{0,5}$, we obtain

$$
\psi=\eta^{2}\left(\tilde{\epsilon}_{1}+\tilde{\epsilon}_{2} \chi+\tilde{\epsilon}_{3} \eta+\frac{\hat{a}_{2,2}}{\hat{a}_{0,5}} \chi^{2}+\tilde{\epsilon}_{4} \chi \eta+\tilde{\epsilon}_{5} \eta^{2}+\eta^{3}+O\left(|\chi, \eta|^{4}\right)\right),
$$

and, finally, to eliminate the $\chi \eta^{2}$ and $\chi \eta^{3}$-terms, we use a transformation

$$
\chi=a+b x_{1}+c y_{1}, \quad \eta=y_{1} .
$$

Choosing the coefficients as

$$
a=-\frac{\tilde{\epsilon}_{2} \hat{a}_{0,5}}{2 \hat{a}_{2,2}}, \quad b=\left|\frac{\hat{a}_{0,5}}{\hat{a}_{2,2}}\right|^{1 / 2}, \quad c=-\frac{\tilde{\epsilon}_{4} \hat{a}_{0,5}}{2 \hat{a}_{2,2}},
$$

the stream function becomes

$$
\psi=y_{1}^{2}\left(c_{0,0}+c_{0,1} y_{1}+c_{0,2} y_{1}^{2}+y_{1}^{3}+\sigma x_{1}^{2}+O\left(\left|x_{1}, y_{1}\right|^{4}\right)\right),
$$

where

$$
c_{0,0}=\epsilon_{1}-\frac{\tilde{\epsilon}_{2}^{2} \hat{a}_{0,5}}{4 \hat{a}_{2,2}}, \quad c_{0,1}=\tilde{\epsilon}_{3}-\frac{\tilde{\epsilon}_{2} \tilde{\epsilon}_{4} \hat{a}_{0,5}}{2 \hat{a}_{2,2}}, \quad c_{0,2}=\tilde{\epsilon}_{5}-\frac{\tilde{\epsilon}_{4}^{2} \hat{a}_{0,5}}{4 \hat{a}_{2,2}}, \quad \sigma=\frac{\hat{a}_{2,2}}{\hat{a}_{0,5}}\left|\frac{\hat{a}_{0,5}}{\hat{a}_{2,2}}\right| .
$$

Note that the definition of $\sigma$ agrees with that of (15) for the degenerate case. When the bifurcation parameters are sufficiently small, $a_{2,2}$ and $\hat{a}_{2,2}$ are of the same sign, as is the case for $\tilde{a}_{0,5}$ and $\hat{a}_{0,5}$. We summarize our findings in the following.

Theorem 1. Let $a_{0,2}, a_{1,2}, a_{0,3}$, and $\tilde{a}_{0,4}$ be small parameters. Assuming the non-degeneracy conditions $a_{2,2} \neq 0$ and $\tilde{a}_{0,5} \neq 0$, a normal form for stream function (17) is

$$
\psi=y^{2}\left(c_{0,0}+c_{0,1} y+c_{0,2} y^{2}+y^{3}+\sigma x^{2}+O\left(|x, y|^{4}\right)\right),
$$

where

$$
\sigma=\left\{\begin{array}{ll}
+1 & \text { for } a_{2,2} / \tilde{a}_{0,5}>0 \\
-1 & \text { for } a_{2,2} / \tilde{a}_{0,5}<0
\end{array},\right.
$$

and $c_{0,0}, c_{0,1}, c_{0,2}$ are transformed small parameters.

\section{Bifurcation analysis of the normal form}

In this section, we analyze the bifurcations in the dynamical system defined by normal form (28). The results are displayed in form of the bifurcation diagrams in Figs. 8-10. The parameter

(a)

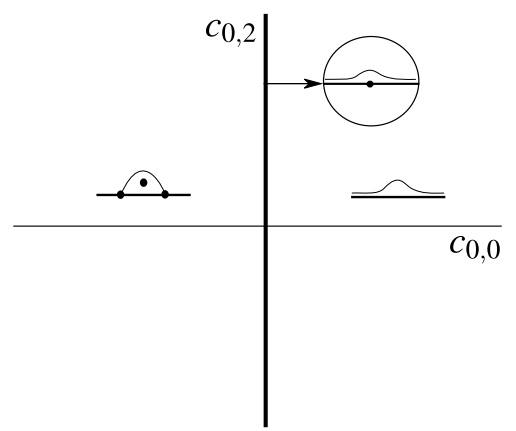

(b)

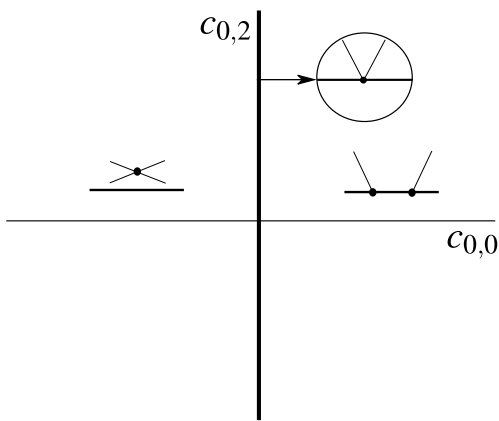

FIG. 8. Bifurcation diagrams for normal form (14) for $\Delta<0$. (a): $\sigma=1$, (b): $\sigma=-1$. 
(a)

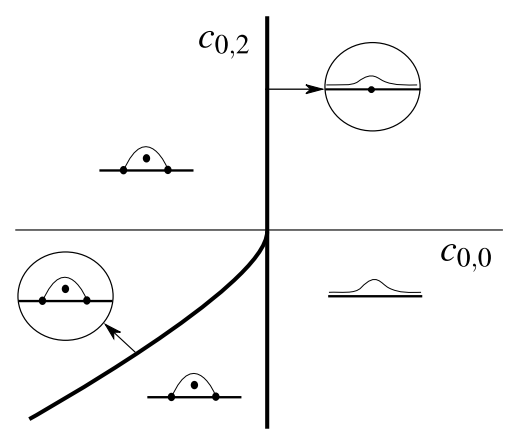

(b)

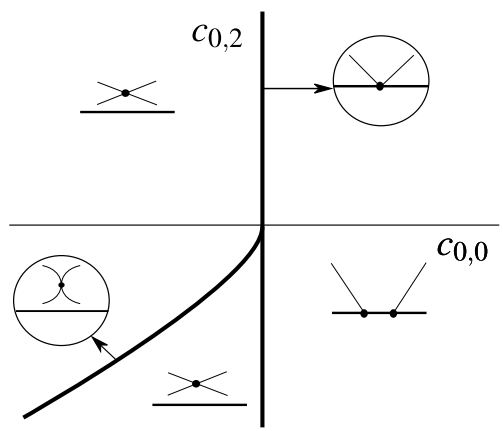

FIG. 9. Bifurcation diagrams for normal form (14) for $\Delta=0$. (a): $\sigma=1$, (b): $\sigma=-1$.

space is three-dimensional, and it turns out to be convenient to use the parameter

$$
\Delta=16 c_{0,2}^{2}-45 c_{0,1}
$$

rather than $c_{0,1}$. Here, we only outline the analysis which is quite involved.

Truncating the $O$-term in (28), the differential equations for the streamlines are, after a scaling of the time by $y$,

$$
\begin{aligned}
& \dot{x}=2 c_{0,0}+3 c_{0,1} y+4 c_{0,2} y^{2}+5 y^{3}+2 \sigma x^{2}, \\
& \dot{y}=-2 \sigma y x,
\end{aligned}
$$

with the determinant of the Jacobian

$$
|J|=6 \sigma c_{0,1} y+16 \sigma c_{0,2} y^{2}-8 x^{2}+30 \sigma y^{3} .
$$

Several types of bifurcation can occur in this system: local bifurcations of on-wall and off-wall critical points; global bifurcations associated with off-wall critical points and global bifurcations

(a)

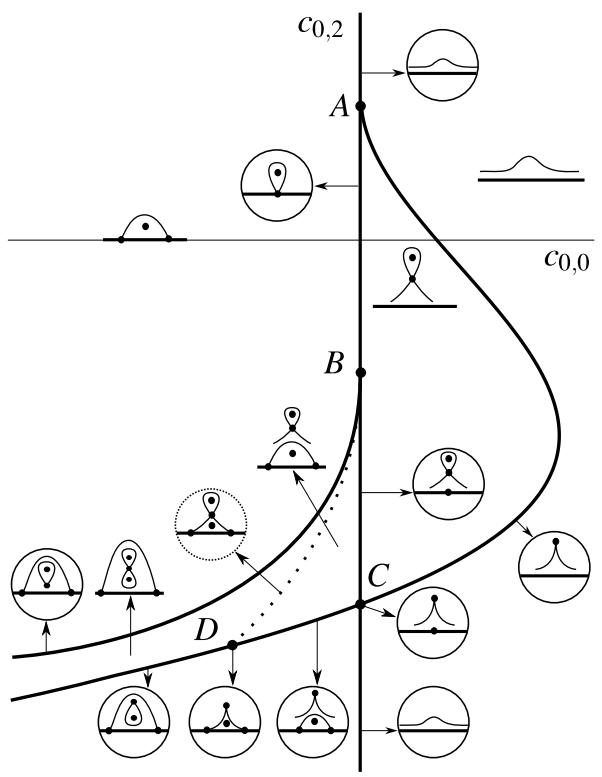

(b)

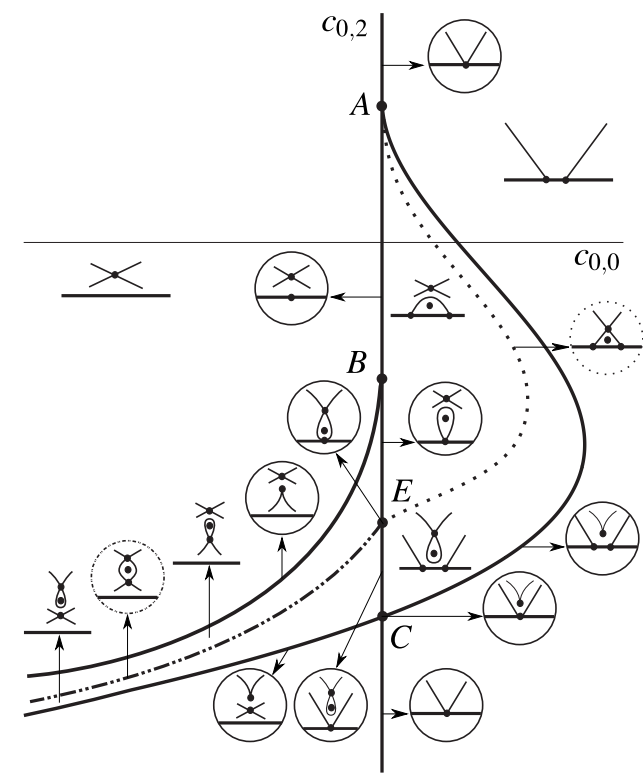

FIG. 10. Bifurcation diagrams for normal form (14) for $\Delta>0$. (a): $\sigma=1$, (b): $\sigma=-1$. The full lines are local bifurcation curves $c_{0,0}=0$ and $\Gamma^{ \pm}$, the dotted lines are the global bifurcation curves $\gamma^{ \pm}$, and the dashed-dotted line is the global bifurcation curve $\zeta^{+}$. The points $A-E$ mark where different bifurcation curves meet. 
associated with on-wall and off-wall critical points. A global bifurcation appears when the value of $\psi$ at different critical points of saddle type coincide. This allows a global (heteroclinic) connection by a dividing streamline from one saddle point to the other. To find local bifurcations of on-wall critical points, we insert $y=0$ into (31a),

$$
\dot{x}=2 c_{0,0}+2 \sigma x^{2}=0 .
$$

From this, one finds that $\pm\left(\sqrt{-\sigma c_{0,0}}, 0\right)$ are critical points. There are two on-wall critical points when $\sigma c_{0,0}<0$, and no critical points when $\sigma c_{0,0}>0$. Hence, a local bifurcation occurs when $c_{0,0}=0$.

To find local bifurcation of off-wall critical points, we substitute $x=0$ into (31a) and (32). Eliminating $y$ from $\dot{x}=0$ and $|J|=0$, one finds

$$
128 c_{0,0} c_{0,2}^{3}-36 c_{0,1}^{2} c_{0,2}^{2}-540 c_{0,0} c_{0,1} c_{0,2}+135 c_{0,1}^{3}+675 c_{0,0}^{2}=0,
$$

where we have removed a factor $c_{0,0}$ which is zero for on-wall bifurcation only. This equation is quadratic in $c_{0,0}$, the discriminant is $\Delta^{3}$, and we find the solution of (34) to be the surfaces in the $\left(c_{0,0}, c_{0,2}, \Delta\right)$ parameter space given by

$$
\Gamma^{ \pm}: c_{0,0}=\frac{32}{675} c_{0,2}{ }^{3}-\frac{2}{225} \Delta c_{0,2} \pm \frac{1}{675} \sqrt{\Delta^{3}},
$$

which are defined only in the half-space $\Delta \geq 0$. Furthermore, only part of these surfaces correspond to bifurcations at points in the fluid domain $y>0$. It can be shown that the bifurcations at $\Gamma^{+}$takes place below the wall for $c_{0,2}>\sqrt{\Delta} / 4$ and similarly for $\Gamma^{-}$for $c_{0,2}>-\sqrt{\Delta} / 4$, and hence these parts do not correspond to a physical bifurcation. The end-points of the bifurcation curves are marked by $A$ and $B$ in Fig. 10.

Proceeding to global bifurcations involving critical points on and off the wall, the condition is that $\psi=0$ at an off-wall critical point fulfilling $\dot{x}=0$ and $x=0$ from (31b). Eliminating $y$ from these equations yields

$$
4 c_{0,0} c_{0,2}^{3}-c_{0,1}^{2} c_{0,2}^{2}-18 c_{0,0} c_{0,1} c_{0,2}+4 c_{0,1}^{3}+27 c_{0,0}^{2}=0,
$$

which again can be solved for $c_{0,0}$,

$$
\gamma^{ \pm}: c_{0,0}=\frac{1}{27} c_{0,2}{ }^{3}-\frac{1}{9} E c_{0,2} \pm \frac{2}{27} \sqrt{E^{3}}
$$

where

$$
E=c_{0,2}^{2}-3 c_{0,1}=\frac{\Delta-c_{0,2}^{2}}{15} .
$$

It follows that the $\gamma^{ \pm}$are defined only when $-\sqrt{\Delta}<c_{0,2}<\sqrt{\Delta}$, and since critical points at the wall must be present, the condition $\sigma c_{0,0}<0$ is also required. Furthermore, the off-wall critical point must be a saddle and hence $|J|<0$. It follows from these inequalities that for $\sigma=1$ only the part $B D$ of $\gamma^{+}$and for $\sigma=-1$, the part $A E$ of $\gamma^{-}$, as shown in Fig. 10, are of significance.

Finally, we consider possible heteroclinic connections among the off-wall critical points. Such connections occur when the stream function attains the same values at two saddle-type critical points $\left(0, y_{1}\right)$ and $\left(0, y_{2}\right)$. The following three conditions must be fulfilled:

$$
\begin{gathered}
\dot{x}\left(0, y_{1}\right)=5 y_{1}{ }^{3}+4 c_{0,2} y_{1}^{2}+3 c_{0,1} y_{1}+2 c_{0,0}=0, \\
\dot{x}\left(0, y_{2}\right)=5 y_{2}{ }^{3}+4 c_{0,2} y_{2}^{2}+3 c_{0,1} y_{2}+2 c_{0,0}=0, \\
\psi\left(0, y_{1}\right)-\psi\left(0, y_{2}\right)=\left(-y_{2}+y_{1}\right) \times \\
\left(c_{0,2} y_{1}^{3}+c_{0,2} y_{1}^{2} y_{2}+c_{0,2} y_{1} y_{2}{ }^{2}+c_{0,2} y_{2}{ }^{3}+y_{1}{ }^{4}+y_{1}{ }^{3} y_{2}+y_{1}{ }^{2} y_{2}{ }^{2}\right. \\
\left.+y_{1} y_{2}{ }^{3}+y_{2}{ }^{4}+c_{0,1} y_{1}{ }^{2}+c_{0,1} y_{1} y_{2}+c_{0,1} y_{2}{ }^{2}+c_{0,0} y_{1}+c_{0,0} y_{2}\right)=0,
\end{gathered}
$$

where $y_{1} \neq y_{2}$. Eliminating $y_{1}$ and $y_{2}$ from these equations gives

$$
128 c_{0,0} c_{0,2}{ }^{3}-36 c_{0,1}^{2} c_{0,2}^{2}-540 c_{0,0} c_{0,1} c_{0,2}+135 c_{0,1}^{3}+675 c_{0,0}^{2}=0,
$$




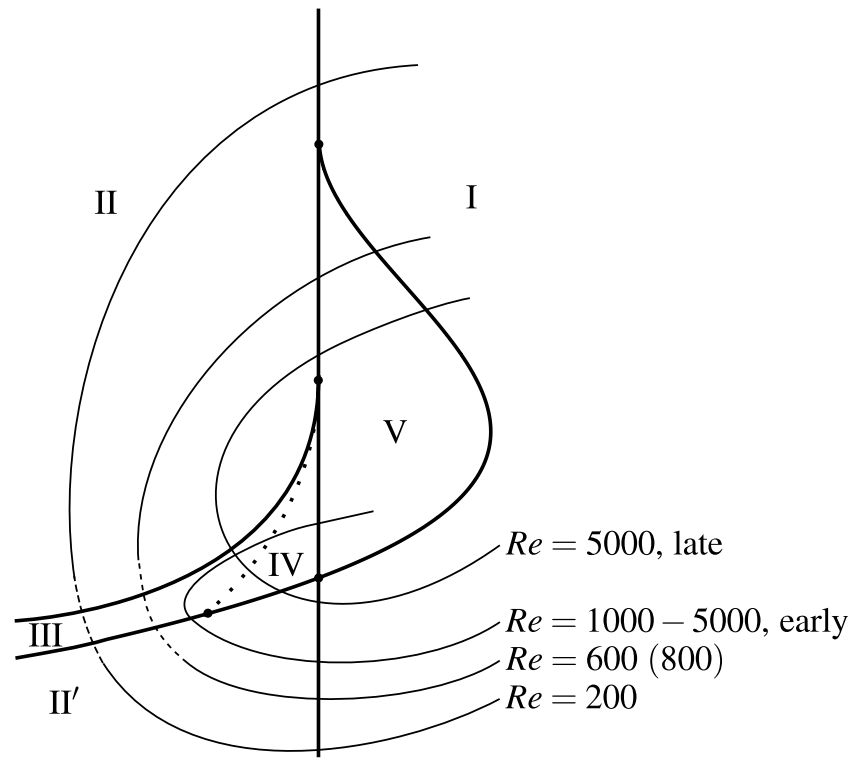

FIG. 11. The bifurcation diagram for normal form (28) for $\sigma=1$. A slice with $\Delta>0$ is shown. The thin lines are time traces for the eruption flow shown in Figs. 2-6. The dashed part represents parts where the path goes through the region with $\Delta<0$. The trace for $R e=800$ is identical to that for $R e=600$ except that the dashed part is a full line such that the path goes through region III.

where we again have removed factors which correspond to bifurcations studied above. Solving for $c_{0,0}$ yields

$$
\zeta^{ \pm}: c_{0,0}=\frac{94}{225} c_{0,2}{ }^{3}-\frac{7}{225} \Delta c_{0,2} \pm \frac{2}{675} \sqrt{15625 c_{0,2}{ }^{6}-1875 \Delta c_{0,2}{ }^{4}+75 \Delta^{2} c_{0,2}{ }^{2}-\Delta^{3}} .
$$

Again, only parts of these surfaces correspond to physical bifurcations. In fact, only the branch of $\zeta^{-}$shown in Fig. 10(b) for $\Delta>0$ and $\sigma=-1$ is relevant.

\section{THE TOPOLOGICAL PHASE SPACE OF ERUPTION}

The bifurcation diagrams obtained in Sec. III D for $\sigma>0$ contains all the bifurcations we have observed in the eruption flow. In Fig. 11, we show a slice in the $\left(c_{0,0}, c_{0,2}, \Delta\right)$ parameter space for $\Delta>0$. The roman numeral in each of the regions is the ones used in Figs. 2-6 to classify the flow topology. Note that the regions II and II' are really one region as they are connected through the part where $\Delta<0$, cf. Fig. 8(a).

Time traces corresponding to the temporal developments observed numerically for the eruption flow are shown in the figure. There are other ways of arranging the paths in accordance with the observations but the one shown is the simplest possible which implies a smooth transition from one path to the next as $R e$ is varied.

Hence, we have obtained a topological phase space for the eruption flow. It accounts for how the streamline patterns are transformed into each other, and it does so in a minimal way: All topologies in the diagram are realized in the flow, and all three dimensions in the parameter space are needed. Thus, it will not be possible to describe the eruption process completely in a simpler topological phase space.

To summarize, the road to primary eruption goes through three stages. The first stage is $R e<$ 600 where only a separation zone on the wall exists. The intermediate stage is $600<R e<1000$ where a small vortex structure is created above the recirculation zone but disappears quickly again. There are different sequences of streamline topologies in this stage, depending on $R e$, and we have not resolved it completely. The final stage is $R e>1000$ where eruption takes place. While we have 
only performed simulations for $R e$ up to 5000 , the results by Kudela and Malecha ${ }^{2}$ indicate that the sequence of topology variations during eruption remains the same for $R e=10000$.

\section{CONCLUSIONS}

Creation and destruction of structures in the streamline pattern of a fluid flow can be classified with a dynamical systems approach. The starting point is a degenerate critical point of the velocity field, and by constructing a bifurcation diagram from an unfolding of the degenerate configuration, a series of possible topological transitions of the flow can be found. The more degenerate the critical point is, the more bifurcation parameters occur in the unfolding, and the more complex patterns can be accounted for. In this paper, we have obtained such bifurcation diagrams for the two-dimensional flow close to a no-slip wall for a degeneracy with co-dimension three. This completes the set of bifurcation diagrams with up to three parameters obtained previously. ${ }^{28,31,32,37,40}$

We have shown that the bifurcation diagram thus obtained in the case $\sigma=1$ describes the basic changes of streamline topology in the eruption process from a boundary layer. The creation of a secondary vortex erupting from the boundary layer is associated with the "pinching off" of a saddle/center pair of the streamlines in a global bifurcation. The bifurcation diagram associated with the singularity we study here is the simplest which can account for both the creation of the recirculation zone on the wall and the pinching off, as no other bifurcation diagram with three parameters or less allows both these transitions. The bifurcation diagrams we have obtained here have at most one secondary erupted vortex. For higher $R e$, several vortices may erupt, and a topological description would require higher-order normal forms for the stream function, occurring as unfoldings of more degenerate critical points. Nevertheless, the transition I $\rightarrow$ II' $\rightarrow$ III $\rightarrow$ IV, shown in Fig. 11 for $R e=5000$, appears to be the fundamental process for the generation of a vortex in the eruption process, and it will occur locally also at much higher Re. As the analysis is topological, and hence of a qualitative nature, it will be valid for a large range of flows, not being critically dependent of the specific physical setting of the eruption process. Thus, the basic degeneracy, given by conditions (8), can be understood as an organizing center for the eruption process.

Other non-simple co-dimension three case (7) plays the same role for vortex shedding behind bluff bodies. In Ref. 40, it is shown how the bifurcation diagram plays a similar role as a topological phase space in the periodic regime where, as in the present paper, distinct dynamical stages in dependence of $R e$ are present.

An alternative approach to study the eruption process is to focus on the topology of the vorticity rather than the stream function. ${ }^{47}$ Simulations ${ }^{2,46}$ clearly indicate that there is a close connection between the flow structures defined by these two fields in the eruption process. A more detailed study of this is in preparation.

Establishing a topological phase space will be of interest in any situation where structures in the flow change as parameters or time vary. The vortex-generated flow studied in the present paper is only an example; in addition to the cases mentioned in the Introduction, another fundamental problem where the method we present would be applicable is the recently studied impulsively started rotation of two cylinders. ${ }^{48}$ Here, topological changes occur in a flow quite different from the one considered here, and, in particular, at much lower Reynolds numbers.

\section{ACKNOWLEDGMENTS}

A.B. is supported by the Turkish Military Academy. M.A. acknowledges the hospitality at Department of Mechanical \& Aerospace Engineering, Monash University, Australia, where part of this work was done.

${ }^{1}$ H. Kudela and Z. M. Malecha, "Investigation of unsteady vorticity layer eruption induced by vortex patch using vortex particles method," J. Theor. Appl. Mech. 45, 785-800 (2007).

${ }^{2}$ H. Kudela and Z. M. Malecha, "Eruption of a boundary layer induced by a 2D vortex patch," Fluid Dyn. Res. 41, 055502 (2009). 
${ }^{3}$ A. V. Obabko and K. W. Cassel, "Navier-Stokes solutions of unsteady separation induced by a vortex," J. Fluid Mech. 465, 99-130 (2002).

${ }^{4}$ J. C. Williams, "Incompressible boundary-layer separation," Annu. Rev. Fluid Mech. 9, 113-144 (1977).

${ }^{5}$ S. K. Robinson, "Coherent motions in the turbulent boundary layer," Annu. Rev. Fluid Mech. 23, 601-639 (1991).

${ }^{6}$ T. L. Doligalski, C. R. Smith, and J. D. A. Walker, "Vortex interactions with walls," Annu. Rev. Fluid Mech. 26, 573-616 (1994).

${ }^{7}$ A. T. Degani, J. D. A. Walker, and F. T. Smith, “Unsteady separation past moving surfaces,” J. Fluid Mech. 375, 1-38 (1998).

${ }^{8}$ J. A. Ekaterinaris and M. F. Platzer, "Computational prediction of airfoil dynamic stall," Prog. Aerosp. Sci. 33, 759-846 (1998).

${ }^{9}$ M. Bross and D. Rockwell, "Flow structure on a simultaneously pitching and rotating wing," J. Fluid Mech. 756, 354-383 (2014).

${ }^{10}$ W.-X. Huang and H. J. Sung, "Vortex shedding from a circular cylinder near a moving wall,” J. Fluids Struct. 23, 1064-1076 (2007).

${ }^{11}$ A. Rao, M. Thompson, T. Leweke, and K. Hourigan, "The flow past a circular cylinder translating at different heights above a wall," J. Fluids Struct. 41, 9-21 (2013).

${ }^{12}$ P. Reichl, K. Hourigan, and M. C. Thompson, "Flow past a cylinder close to a free surface," J. Fluid Mech. 533, 269-296 (2005).

${ }^{13}$ M. Brøns, M. C. Thompson, T. Leweke, and K. Hourigan, "Vorticity generation and conservation for two-dimensional interfaces and boundaries," J. Fluid Mech. 758, 63-93 (2014).

${ }^{14}$ P. Orlandi, "Vortex dipole rebound from a wall," Phys. Fluids A 2, 1429-1436 (1990).

${ }^{15}$ D. Sutherland, C. Macaskill, and D. G. Dritschel, "The effect of slip length on vortex rebound from a rigid boundary," Phys. Fluids 25, 093104 (2013).

${ }^{16}$ M. C. Thompson, T. Leweke, and K. Hourigan, "Sphere-wall collisions: Vortex dynamics and stability," J. Fluid Mech. 575, 121-148 (2007).

${ }^{17}$ N. Didden and C.-M. Ho, "Unsteady separation in a boundary layer produced by an impinging jet," J. Fluid Mech. 160, 235-256 (1985).

${ }^{18}$ H. J. Lugt, "The dilemma of defining a vortex," in Recent Developments in Theoretical and Experimental Fluid Mechanics, edited by U. Müller, K. G. Roesner, and B. Schmidt (Springer, 1979), pp. 309-321.

${ }^{19}$ J. Jeong and F. Hussain, "On the identification of a vortex," J. Fluid Mech. 285, 69-94 (1995).

${ }^{20}$ G. Haller, "An objective definition of a vortex," J. Fluid Mech. 525, 1-26 (2005).

${ }^{21}$ W. R. Dean, "Note on the motion of liquid near a position of separation," Math. Proc. Cambridge Philos. Soc. 46, 293-306 (1950).

${ }^{22}$ R. Legendre, "Seperation de l'ecoulement laminaire tridimensionel," La Recherche Aérospatiale 54, 3-8 (1958).

${ }^{23}$ R. Legendre, "Lignes de courant d'un ecoulement continu," La Recherche Aérospatiale 105, 3-9 (1965).

${ }^{24}$ J. Délery, "Robert Legendre and Henri Werlé: Toward the elucidation of three-dimensional separation," Annu. Rev. Fluid Mech. 33, 129-154 (2001)

${ }^{25}$ M. Tobak and D. J. Peake, "Topology of three-dimensional separated flows," Annu. Rev. Fluid Mech. 14, 61-85 (1982).

${ }^{26}$ A. E. Perry and H. Hornung, "Some aspects of three-dimensional separation, part II: Vortex skeletons," Zeitschrift für Flugwissenshaft und Weltraumforschung 8, 155-160 (1984).

${ }^{27}$ A. E. Perry and M. S. Chong, "A description of eddying motions and flow patterns using critical-point concepts," Annu. Rev. Fluid Mech. 19, 125-155 (1987).

${ }^{28}$ P. G. Bakker, Bifurcations in Flow Patterns (Klüver Academic Publishers, Dordrecht, 1991).

${ }^{29}$ M. Brøns, "Topological fluid dynamics of interfacial flows," Phys. Fluids 6, 2730-2737 (1994).

${ }^{30}$ M. Brøns and J. N. Hartnack, "Streamline topologies near simple degenerate critical points in two-dimensional flow away from boundaries," Phys. Fluids 11, 314-324 (1999).

31 J. N. Hartnack, "Streamline topologies near a fixed wall using normal forms," Acta Mech. 136, 55-75 (1999).

${ }^{32}$ M. Brøns, "Streamline topology: Patterns in fluid flows and their bifurcations," Adv. Appl. Mech. 41, 1-42 (2007).

${ }^{33}$ A. Deliceoğlu, "Topology of two-dimensional flow associated with degenerate dividing streamline on a free surface," Eur. J. Appl. Math. 24, 77-101 (2013).

${ }^{34}$ M. Brøns, L. K. Voigt, and J. N. Sørensen, "Streamline topology of steady axisymmetric vortex breakdown in a cylinder with co- and counter-rotating end-covers," J. Fluid Mech. 401, 275-292 (1999).

${ }^{35}$ M. Brøns, L. K. Voigt, and J. N. Sørensen, "Topology of vortex breakdown bubbles in a cylinder with a rotating bottom and a free surface,” J. Fluid Mech. 428, 133-148 (2001).

${ }^{36}$ F. Gürcan and A. Deliceoğlu, "Streamline topologies near nonsimple degenerate points in two-dimensional flows with double symmetry away from boundaries and an application," Phys. Fluids 17, 093106 (2005).

${ }^{37}$ F. Gürcan, A. Deliceoğlu, and P. G. Bakker, "Streamline topologies near a non-simple degenerate critical point close to a stationary wall using normal forms," J. Fluid Mech. 539, 299-311 (2005).

${ }^{38}$ F. Gürcan and A. Deliceoğlu, "Saddle connections near degenerate critical points in Stokes flow within cavities," Appl. Math. Comput. 172, 1133-1144 (2006).

${ }^{39}$ A. E. Perry, M. S. Chong, and T. T. Lim, “The vortex-shedding process behind two-dimensional bluff bodies,” J. Fluid Mech. 116, 77-90 (1982).

${ }^{40}$ M. Brøns, B. Jakobsen, K. Niss, A. V. Bisgaard, and L. K. Voigt, "Streamline topology in the near wake of a circular cylinder at moderate Reynolds numbers," J. Fluid Mech. 584, 23-43 (2007).

${ }^{41}$ J. Jiménez-Lozano and M. Sen, "Streamline topologies of two-dimensional peristaltic flow and their bifurcations," Chem. Eng. Process.: Process Intensif. 49, 704-715 (2010).

${ }^{42}$ C. Roy, N. Schaeffer, S. L. Dizès, and M. Thompson, "Stability of a pair of co-rotating vortices with axial flow," Phys. Fluids 20, 094101 (2008). 
${ }^{43}$ G. E. Karniadakis, M. Israeli, and S. A. Orszag, "High-order splitting methods for the incompressible Navier-Stokes equations,” J. Comput. Phys. 97, 414-443 (1991).

${ }^{44}$ G. E. Karniadakis and S. Sherwin, Spectral/hp Element Methods for Computational Fluid Dynamics, 2nd ed. (Oxford University Press, 2005).

${ }^{45}$ M. C. Thompson, K. Hourigan, A. Cheung, and T. Leweke, "Hydrodynamics of a particle impact on a wall," Appl. Math. Modell. 30(11), 1356-1369 (2006).

${ }^{46} \mathrm{M}$. Andersen, "Topology of streamlines and vorticity contours for two-dimensional flows," Ph.D. thesis (Department of Applied Mathematics and Computer Science, Technical University of Denmark, 2013).

${ }^{47}$ M. Brøns and A. V. Bisgaard, "Topology of vortex creation in the cylinder wake," Theor. Comput. Fluid Dyn. 24, 299-303 (2010).

${ }^{48}$ Y. Ueda, T. Kida, and M. Iguchi, "Steady approach of unsteady low-Reynolds-number flow past two rotating circular cylinders," J. Fluid Mech. 736, 414-443 (2013). 